\title{
Control of Traffic Light in Isolated Intersections Using Fuzzy Neural Network and Genetic Algorithm
}

\author{
T. Royani, J. Haddadnia, and M. Alipoor
}

\begin{abstract}
In this paper a fuzzy neural network is applied for real time traffic signal control at an isolated intersection. The FNN has advantages of both fuzzy expert system (fuzzy reasoning) and artificial neural network (self-study).

A traffic light controller based on fuzzy neural network can be used for optimum control of fluctuating traffic volumes such as oversaturated or unusual load condition. The objective is to improve the vehicular throughput and minimize delays. The rules of fuzzy logic controller are formulated by following the same protocols that a human operator would use to control the time intervals of the traffic light. For adjusting the parameters of FNN, genetic algorithm was used. Compared with traditional control methods for traffic signal, the proposed FNN algorithm shows better performance and adaptability.
\end{abstract}

Index Terms-Fuzzy neural network, traffic control, delay, genetic algorithm, performance.

\section{INTRODUCTION}

Traffic congestion is a crucial problem in large cities. Signal control methods include traditional control methods and intelligent control methods. Since intelligent control methods are superior to traditional control methods, lots of intelligent signal control models were put forward in recent years. It is partially caused by improper control of traffic lights, which is not corresponding to the current traffic conditions. To alleviate traffic congestion in urban areas, the concept of Intelligent Transportation Systems (ITS) has been widely accepted in developed countries. ITS is a highly promising system for providing key solutions to current road congestion problems [1].

The problem of intelligent traffic control has been studied in the area of ITS for many years. There are many conventional methods for traffic signal control but most of them sometimes fail to deal efficiently with the complex, time-varying traffic conditions and controller can't satisfy real-time character for traffic signal [2]. They are modeled based on the preset cycle time to change the signal without any analysis of traffic situation. Due to fixed cycle time, such systems do not consider that which intersection has more load of traffic, so should kept green more or should terminate earlier then complete cycle time. Recently, major research on urban traffic focuses on artificial intelligence techniques, such as fuzzy control, genetic algorithm and neural network.

Manuscript received October 10, 2012; reviesed November 15, 2012

T. Royani and J. Haddadnia are with Tarbiat Moallem University of Sabzevar, Electrical and Computer Engineering Faculty, Sabzevar, Iran (e-mail: tahere.royani@gmail.com; haddadnia@sttu.ac.ir).

M. Alipoor was with Tarbiat Moallem University of Sabzevar, Electrical and Computer Engineering Faculty, Sabzevar, Iran. He is now with Chalmers University of Technology, Sweden (e-mail: mbiomedical@yahoo.com).
Using timed Petri Nets [3], SPSA [4], ant algorithm [5], knowledge based multi-agent system [6], [7] and a mobile agent [8] have also been suggested.

Trabia [9] designed a multi-phase fuzzy logic controller for an isolated intersection with through and left-turning movements. In [10], a new fuzzy controller based on fuzzy logic and weighting coefficients is designed. Bingham [11] obtained intersection fuzzy control parameters from neural networks, and improved fuzzy control result. Chen Xiangjun [12] put forward a self-learning traffic signal control approach, which controls intersection signal with fuzzy algorithm, and updates fuzzy control rules with genetic algorithm.

These studies have their own characteristics and theoretical foundations; however, an intersection signal control model should consider three factors:

1) Simplified computing model, control schemes should output in a specified period; 2) consider both under control intersection and its adjacent intersection, for realizing linear or group control; 3) self-learning ability. This paper tries to consider these factors in intersection control model. Through fuzzy classifying traffic flow in under control intersection, the model save signal control schemes in different traffic flow into knowledge-database as rule set. In control process, the model use neural network to update rule set according to different control effect of control schemes in different traffic flow, thus the model has self-learning ability [13]. The rules of fuzzy logic controller are formulated by following the same protocols that a human operator would use to control the time intervals of the traffic light. The length of current green phase is extended or terminated depending upon the "arrival", number of vehicle approaching the green phase and "queue" that corresponds to the number of queuing vehicles in red or green phases.

By using MATLAB tool for simulation and experiments, it proves that model control effect is obviously superior to traditional control methods.

The paper is organized as follows:

Section II deals with basic of fuzzy logic systems, rule bases and membership functions. The fuzzy neural network model for traffic signal control at the isolated intersection and proposed method are explained in section III. Experimented and analyses are shown in section IV. Section V describes conclusions.

\section{FUZZY LOGIC SYSTEMS FOR TRAFFIC SIGNAL CONTROL}

Fuzzy logic is a distinct idea for developing models of physical processes. Fuzzy models are less externally complex; they can be understood easily and very much suitable for 
non-linear processes. Models with fewer rules are more advantageous. Fuzzy controllers have the ability to take decision even with incomplete information. More and more sophisticated fuzzy logic controllers are being developed for traffic control [14]-[21]. These algorithms are continually improving the safety and efficiency by reducing the waiting delay of vehicles on signals [22].

Fuzzy logic allows linguistic and inexact data to be manipulated as a useful tool in designing signal timings. Also the linguistic control strategy that is decided by "if-then-else" statement can be converted in to a control algorithm using fuzzy logic. The design of a fuzzy signal controller needs an expert knowledge and experience of traffic control in formulating the linguistic protocol, which generates the control input to the traffic signal control system.

The input variables of the fuzzy controller in this paper are designed as follow; one is the number of approaching vehicles in the current green phase (denoted by $A v_{i}$ ); the another is the number of queuing vehicles in the current green phase (denoted by $\mathrm{qg}_{\mathrm{i}}$ ) and the third one is the number of queuing vehicles in the current red phase (denoted by $\mathrm{qr}_{\mathrm{i}}$ ). Here, $\mathrm{i}$ refers to the sequence number of the signal current phase. The output variable is the extended time in the current green phase (expressed by $\Delta \mathrm{t}_{\mathrm{i}}$ ). Suppose for this work that $\mathrm{AV}_{\mathrm{i}}$ take on following linguistic values: L(low), M(medium), $\mathrm{H}$ (high); and $\mathrm{qg}_{\mathrm{i}}, \mathrm{qr}_{\mathrm{i}}$ take on the following linguistic values: $\mathrm{S}$ (short), $\mathrm{M}$ (medium), L(long), $\mathrm{VL}$ (very long). $\Delta \mathrm{t}_{\mathrm{i}}$ take on $\mathrm{Z}$ (zero), S(short), M(medium), L(long). The fuzzy sets of input and output variables are shown in Fig. 1. For this inputs we have 48 fuzzy rules that some of them are shown in Table I.

We know that the maximum of phase extension is " 30 s" and maximum of queue is 90 and Initialize the phases minimal time "tmin $=15 \mathrm{~s}$ ".
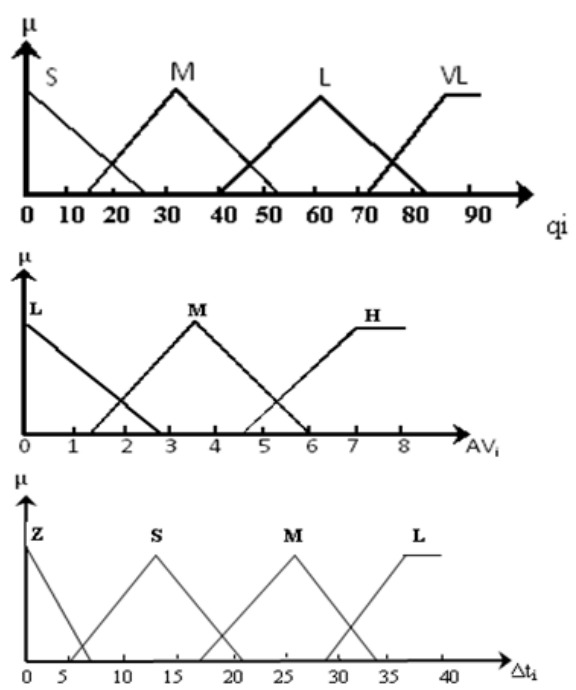

Fig. 1. Membership function of inputs and output.

TABLE I: FUZZY RULES OF TRAFFIC CONTROL SYSTEM

If $\mathrm{Qg}$ is short and Qr is short and AV is low Then $\Delta \mathrm{t}$ is short.

If $\mathrm{Qg}$ is long and Qr is medium and AV is medium Then $\Delta \mathrm{t}$ is short.

If $\mathrm{Qg}$ is medium and $\mathrm{Qr}$ is long and $\mathrm{AV}$ is low Then $\Delta \mathrm{t}$ is zero.

If $\mathrm{Qg}$ is medium and $\mathrm{Qr}$ is very long and $\mathrm{AV}$ is high Then $\Delta \mathrm{t}$ zero.

If $\mathrm{Qg}$ is very long and $\mathrm{Qr}$ is short and $\mathrm{AV}$ is low Then $\Delta \mathrm{t}$ is long.

If $\mathrm{Qg}$ is short and $\mathrm{Qr}$ is very long and $\mathrm{AV}$ is high Then $\Delta \mathrm{t}$ is zero.

\section{PROPOSED METHOD}

\section{A. Fuzzy Neural Network Modeling}

Artificial neural networks (ANNs) (learning systems) and expert system (knowledge-based systems) have been extensively explored as approaches for decision making. While the ANNs compute decisions by learning from successfully solved examples, the expert systems rely on a knowledge base developed by human reasoning for decision making. An important aspect in intelligent system design is decision explanation, which involves supplying a coherent explanation of its decisions [2]. This is required for 1) acceptability of the solution and 2) correctness of the reasoning process by evaluating the trace generated by the inference engine or by analyzing the rule base (which typically use "IF THEN" rules). Also, in learning system such as ANNs, knowledge is represented in the form of weighted connections, making decision tracing or extraction difficult.

Therefore by using an ANN or an expert system approach to intelligent decision making leads to different levels of performance depending on the model as well as the application. By integrating the two approaches, it is possible to overcome the deficiencies associated with using a single approach. The key properties of neuro-fuzzy systems are the accurate learning and adaptive capabilities of the neural networks, together with the generalization and fast-learning capabilities of fuzzy logic systems [2]. The fuzzy neural network that is used in our work is a five-layer dedicated neural network, as shown in Fig. 2, designed according to the working process of fuzzy controller systems; it was presented in [2]. The relation and functions of the nodes in the network are as follows.

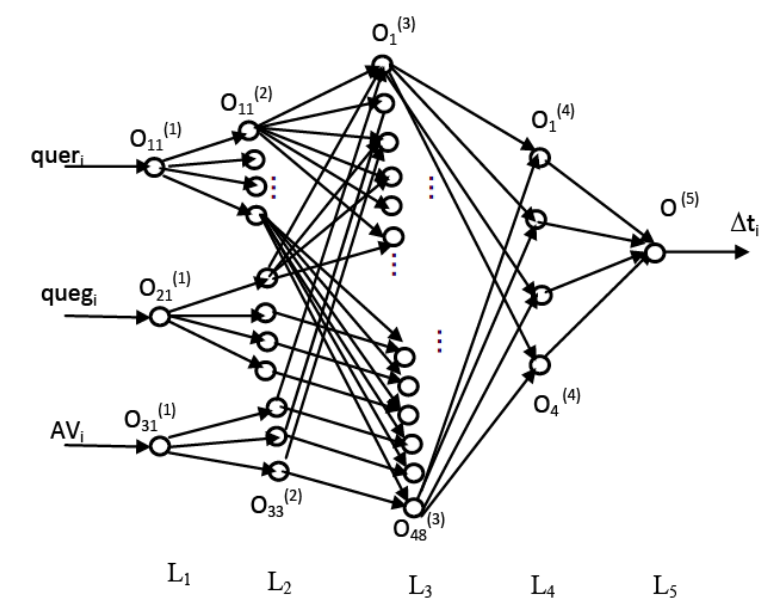

Fig. 2. Model of fuzzy neural network

The first layer is input layer; the nodes represent input linguistic variables of quer ${ }_{i}$, queg $_{i}$ and $A V_{i}$. So the outputs of first layer can be written as

$$
\mathrm{O}_{11}{ }^{(1)}=\mathrm{qr}_{\mathrm{i}}, \mathrm{O}_{21}{ }^{(1)}=\mathrm{qg}_{\mathrm{i}}, \mathrm{O}_{31}{ }^{(1)}=\mathrm{AV}_{\mathrm{i}}
$$

The second layer is membership-function layer. Each node in this layer represents the membership function of a linguistic value associated with an input linguistic variable. 
The output of each node is in the range $[0,1]$ and represents the membership grade of the input with respect to the membership function. Here, Gauss function is used to carve up the input signal. The output of each node is:

$$
O_{j k}^{(2)}=\exp { }^{\left(-\frac{\left(O_{j k}^{(1)}-a_{j k}\right)^{2}}{b_{j k}^{2}}\right)}
$$

where $j=1,2 ; k=1,2, \ldots, 4$; and for $j=3 ; k=1,2,3$; ajk and bjk are parameters of centroid and width respectively. These parameters will be adjusted in back propagation step.

The third layer of the fuzzy controller network corresponds to the rule base. A fuzzy rule is characterized by the relationship between the antecedent and the consequent, and an action that is inferred from a set of fuzzy rules and a fuzzy relation composed from the rules. If $\mathrm{A}, \mathrm{B}$ and $\mathrm{C}$ are fuzzy subsets representing linguistic variables, then a decision from a rule "if $A \& B$ then $C$ " is defined by the membership functions on $\mathrm{A}$ and $\mathrm{B}$ and a node $\mathrm{p}$ in the third layer computes the rule firing strength, In our network, the "and" combiner in the rules is interpreted as the minimum operator suggested by Zadeh [23]. By the way the degree to which rule $p$ is satisfied, result of this layer can be represents as

$$
\begin{gathered}
\mathrm{O}_{\mathrm{p}}^{(3)=} \operatorname{Min}\left(\mathrm{O}_{\mathrm{jk}}{ }^{(2)}\right), \mathrm{p}=1,2, \ldots, 48 \text { or } \\
\mathrm{O}_{\mathrm{p}}{ }^{(3)}=\operatorname{Min}\left(\mu_{\mathrm{qr}}, \mu_{\mathrm{qg}}, \mu_{\mathrm{AV}}\right)
\end{gathered}
$$

Refer to "(3)," $p$ is the number of rules; we have "48 rules" in our work.

The forth layer of the fuzzy controller network corresponds to the rule consequents. Initially, the links between the third and fourth layer are fully connected so that all the possible fuzzy rules are embedded in the structure of the network. The weight $\alpha_{p}(1 \leq p \leq 48)$ of an input link in the layer represents the certainty factor of a fuzzy rules. Hence, these weights are adjustable while learning the knowledge of fuzzy rules. We choose the max-operator suggested by Zadeh [23] as the function of a node in the layer. With this explanation the outputs of this layer are

$$
\mathrm{O}_{\mathrm{j}}^{(4)}=\operatorname{Max}\left(\alpha_{\mathrm{p}} \cdot \mathrm{O}_{\mathrm{p}}^{(3)}\right), \mathrm{j}=1, \ldots, 4
$$

The fifth layer is defuzzification layer. The node in this layer represents the output linguistic variable and performs defuzzification, taking into consideration the effects of all the membership functions of the linguistic values of the output. We choose the correlation-product inference and the fuzzy centroid defuzzification scheme, and then the function of the output node is defined as

$$
O^{(5)}=\sum_{j=1}^{4}\left(O_{j}^{(4)} a_{j} b_{j}\right) / \sum_{j=1}^{4}\left(O_{j}^{4} a_{j}\right)
$$

where $a_{\mathrm{j}}$ and $b_{\mathrm{j}}$ are the area and centroid of the membership function of the output linguistic value respectively. Since it is assumed that the membership functions of the output linguistic values are known, the areas and centroids can be calculated before learning.

\section{B. Training the FNN Network with Genetic Algorithm}

The error gradient descent is chosen as the training algorithm. In the training phase, the concept of genetic algorithm is used to minimize the least mean square (LMS) error function:

$$
E(\vec{w}) \equiv \frac{1}{2} \sum_{d \in D} \sum_{k \in \text { outputs }}\left(t_{k d}-o_{k d}\right)^{2}
$$

$E$ defined as a sum of the squared errors over all the output $\mathrm{k}$ units for all the training examples $\mathrm{d}$.

$$
E_{i}=\left(t_{i}-O^{(5)}\right)^{2} / 2
$$

Refer to "(7)," $\mathrm{t}_{\mathrm{i}}$ is the our target for $i^{\text {th }}$ input, and $O^{(5)}$ is output of our network. For adjusting parameters in learning process, we use genetic algorithm instead of back propagation method, because BP for this network is very complicated and suffer from computational complexity. A genetic algorithm maintains a population of candidate solutions for the problem at hand, and makes it evolve by iteratively applying a set of stochastic operators. Genetic algorithm attempts to minimize the squared error between the network output values and the target values for these outputs. The weight update loop may be iterated thousands of times in a typical application.

Construct the training algorithm:

Step1: Input the parameters of the control algorithm and the sample value to train.

Step2: define squared error as fitness function of GA and FNN weights as its variables.

Step 3: Produce an initial population of individuals

Step 4: Evaluate the fitness of all individuals

Select fitter individuals for reproduction

Recombine between individuals

Mutate individuals

Evaluate the fitness of the modified individuals

Generate a new population

Step5: If termination condition met and the trained error is less than the demanded trained error, then the train of the network is end and the weights are outputted, else return to step 4 and continue to train the network.

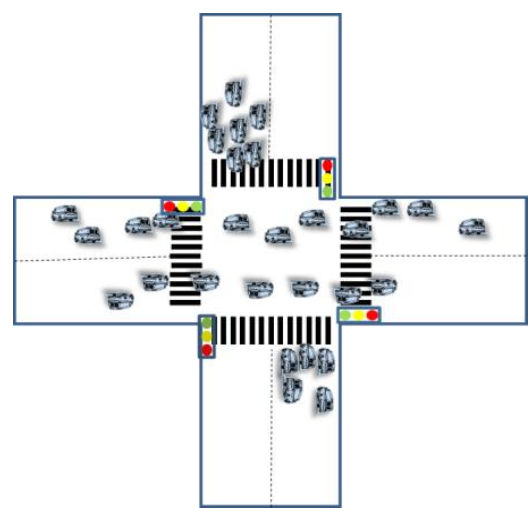

Fig. 3. Model of an isolated intersection 


\section{Simulation AND Results}

\section{A. Model of an Intersection}

An isolated signalized intersection with four legs and three lanes in each leg is studied in this paper. Fig. 3 shows a typical intersection with lanes and vehicle detectors configuration. Each approach has straight movement without left-turning and right-turning. Four cameras for vehicle detection are installed on stop-lines. The cameras provide vehicles' information and number of vehicles waiting in the lanes. Traffic signal is controlled by two phases. Minimum of cycle is about " $40 \mathrm{~s}$ " and maximum of cycle is about " $120 \mathrm{~s}$ ".

\section{B. Simulation}

The simulation is carried out using MATLAB 7.8 and the Genetic Algorithm Toolbox. The Genetic Algorithm Toolbox is useful to train quickly the network and changes are easily made. This significantly reduces the development time of the simulation model. The new fuzzy neural network traffic controller can optimally control traffic flows under both normal and abnormal traffic conditions. The criterion of optimization is the decrement length of queues and the average of waiting time vehicles in intersection. Our samples are real and extracted from traffic video sequences. Sampling time is " $10 \mathrm{~s}$ ". The minimum green time $\left(\mathrm{g}_{\min }\right)$ is preset as " 10 $s$ " in order to let the vehicles cross the intersection safely; the maximum of the extension of green time $\left(\mathrm{e}_{\max }\right)$ is " $30 \mathrm{~s}$ ". Pedestrians are not considered in this study.

The parameters of this GA are set as: population size $=220$, crossover probability $=0.5$, mutation probability $=0.01$.

The stop condition is ( $f i+1-f i) \leq \varepsilon$ where $f_{i}$ is the maximum fitness function among the population for the $\mathrm{s}^{\text {th }}$ iterative evolution and $\varepsilon$ is 0.01 .

\section{Results}

For testing our traffic control system, we use it at a simulated intersection in MALAB with real situation. We compare its results with fixed-time control system. The results of simulation for fixed-time control and Fuzzy Neural Network control were demonstrated in Fig. 4 and Fig. 5. Table II shows the results of waiting time for fixed-time control and fuzzy intelligent control for 1000 vehicles in four lanes of intersection.

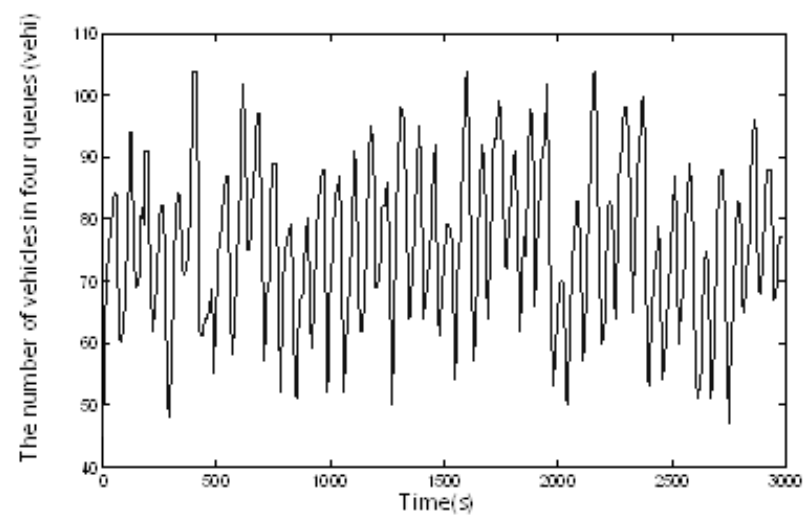

Fig 4. The number of vehicles in. four queues of intersection without controller.

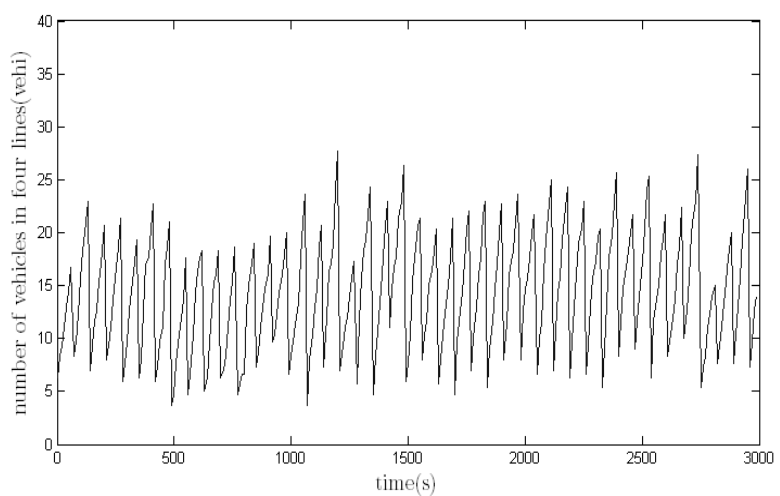

Fig. 5. The number of vehicles in four queues of intersection with FNN controller.

TABLE II: WAITING TIME COMPARISON FOR 1000 VEHICLES

\begin{tabular}{cc}
\hline \hline Control Method & Average Waiting Time(second) \\
\hline Fixed Time Control & $330 \mathrm{~s}$ \\
FNN control & $178 \mathrm{~s}$ \\
\hline \hline
\end{tabular}

\section{CONCLUSION}

In this paper we attempted to apply the Fuzzy Neural Network model to traffic signal controller with expert knowledge. The fuzzy neural network is tuned by the proposed learning method. We use genetic algorithm for learning process. By the learning of the neural network, we can tune the fuzzy model and optimize system's parameters. The research results have proved feasibility and validity of the proposed FNN algorithm.

\section{REFERENCES}

[1] F. Daneshfar and J. RavanJamJah, "Adaptive Fuzzy Urban Traffic Flow Control Using a Cooperative Multi-Agent System based on Two Stage Fuzzy Clustering," IEEE Trans.

[2] L.Zang, L. Jia, and Y. Luo, "An Intelligent Control Method for Urban Traffic Signal Based on Fuzzy Neural Network," in Proc. 6th World Congress on Intelligent Control and Automation, 2006, Dalian, China.

[3] A. Th. Van den Bosch, M. R. Menken, M. V. Breukelen, and R. T.Van Katwijk, "A test bed for multi-agent system and road traffic management," in Proc. 15th Belgian-Netherlands Conf. on Artificial Intelligence (BNAI'03), Vrije Universiteit, Amsterdam, 2003.

[4] M. C. Choy, D. Srinivasan, and R. L. Cheu, "Simultaneous perturbation stochastic approximation based neural networks for online learning," in Proc. 7th IEEE Int. Conf. Intelligent Transportation Systems, October 2004, pp. 1038-1044.

[5] C. Bertelle, A. Dutot, S. Lerebourg, and D. Olivier, "Road traffic management based on ant system and regulation model," in Proc. MAS conference, 2003.URL: http://www.liophant.org/mas2003/index.html

[6] K. Dresner and P. Stone, "Multiagent traffic management: an improved intersection control mechanism," in Proc. $5^{\text {th }}$ AAMAS, July 2529, 2005 , Utrecht, the Netherlands.

[7] V. Hirankitti and J. Krohkaew, "An agent approach for intelligent traffic-light control," in Proc. First Asia International Conference on Modeling \& Simulation (AMS'07), pp. 496-501, 2007.

[8] H. Kheyruri, A. Z. Jahangiri, and A. Pardakhtchi, "Independent agents for urban traffic control problem with mobile-agent coordination," in Proc. 7th WSEAS International Conf. Computer Science, Venice, Italy, 2007, pp. 372-376.

[9] M. B. Trabia, M. S. Kaseko, and M. Ande, "A twostage fuzzy logic controller for traffic signals," Transportation Research Part C, pp. 353-367, 1999.

[10] Y. Li and X. Fan, "Design of signal controllers for urban intersections based on fuzzy logic and weightings," IEEE Conference, vol.1, pp.867 $-871,2003$.

[11] E. Bingham, "Reinforcement learning in neurofuzzy traffic signal control," European Journal of Operational Research, vol.131, no.1, pp. 232-241. 
[12] C. Xiangjun and Y. Zhaoxia "A Self-learning Traffic Signal Control Approach and Simulation," Journal of System Simulation, vol. 16, no. 7, pp. 1519-1524, 2004.

[13] Y. Wei, Q. Shao, Y. Han, and B. Fan, "Intersection Signal Control Approach based on PSO and Simulation," in Proc. Second International Conference on Genetic and Evolutionary Computing, 2008 IEEE.

[14] C. P. Pappis and E. H. Mamdani, "A Fuzzy Logic Controller for a Traffic Junction," IEEE Transactions on Systems, Man, and Cybernetics, vol. SMC-7, no. 10, October 1977, pp. 707-717.

[15] R. L.Kelsey and K. R. Bisset, "Simulation of Traffic Flow and Control Using Fuzzy and Conventional Methods," Fuzzy Logic and Control:Software and Hardware Applications, Prentice Hall, Englewood Cliffs, New Jersey, 1993, pp.262-278.

[16] J. Niittymaki and S. Kikuchi, "Application of Fuzzy Logic to the Control of a Pedestrian Crossing Signal," in Transportation Research Record: Journal of the Transportation Research Board, no. 1651, TRB, National Research Council, Washington, D.C., 1998, pp. 30-38.

[17] S. Chiu, "Adaptive Traffic Signal Control Using Fuzzy Logic," in Proc. IEEE Intelligent Vehicles Symposium, 1992, pp. 98-107.

[18] J. Niittymaki and M. Pursula, "Signal Control Using Fuzzy Logic," Fuzzy Sets and Systems, vol. 116, 2000, pp. 11-22.

[19] M. Nakatsuyama, H. Nagahashi, and N. Nishizuka, "Fuzzy Logic Phase Controller for Traffic Junctions in the One-Way Arterial Road," in Proc. IFAC Ninth Triennial World Congress, 1984, pp. 2865-2870.

[20] H. Li, P. D. Prevedouros, and L. Zhang, Signal Control for Oversaturated Intersections Using Fuzzy Logic, presented at the 2005 Annual Meeting of the TRB.

[21] H. Wei, W. Yong, M. Xuanqin, and W. Yan, "A cooperative fuzzy control method for traffic Lights," in Proc. Intelligent Transportation Systems Conference, Oakland (CA), USA - August 25-29, 2001.

[22] J. Niittymäki and M. Mäenpää. "The role of fuzzy logic public transport priority in traffic signal control," Traffic Engineering and Control, International Journal of Traffic Management and Transportation Planning, January 2001, Hemming-Group Ltd. By permission.

[23] L. A. Zadeh, "Fuzzy sets," Inform. And Control, vol. 8, pp. 338-353, 1965.

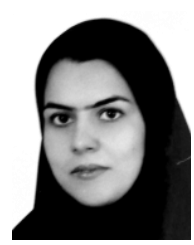

Tahere Royani was born in Iran, in 1984. He received the B.Sc. degree in electrical engineering from Ferdowsi University of Mashhad, Iran in 2007 and M.Sc. degree from Tarbiat Moallem University of Sabzevar, Iran in 2010, respectively. She is the author or coauthor of more than five national and international papers. Her current research interests include Fuzzy Systems, Signal and Image processing.

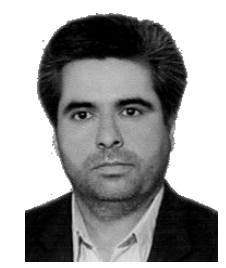

Javad Haddadnia received his B.S. and M.S. degrees in electrical and electronic engineering with the first rank from Amirkabir University of Technology, Tehran, Iran, in 1993 and 1995, respectively. He received his Ph.D. degree in electrical engineering from Amirkabir University of Technology, Tehran, Iran in 2002. He joined Tarbiat Moallem University of Sabzevar in Iran. His research interests include neural network, digital image processing, computer vision, and face detection and recognition. He has published several papers in these areas. He has served as a Visiting Research Scholar at the University of Windsor, Canada during 2001 and 2002. He is a member of SPIE, CIPPR, and IEICE.

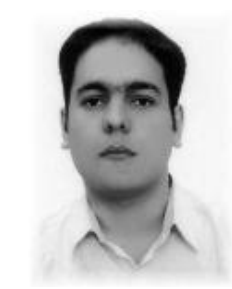

Mohammad Alipoor was born in Tabriz, Iran, in 1984. He received the B.S. degree in bioelectrical engineering from Sahand University of Tabriz, Iran in 2006, and obtained the M.S. degree in electronic from Tarbiat Moallem University Of Sabzevar, Iran in 2009. He is currently pursuing his Ph.D. degree in Chalmers University of Technology, Sweden. He is the author or coauthor of more than ten national and international papers. His current research interests include fuzzy systems, analysis of biomedical signals, signal and image processing. 